influx of shallow-water carbonate debris, a rapid reduction in the amount of pelagic carbonate and a period of slow or condensed sedimentation; it is followed by the onset of accumulation of carbonatepoor bioturbated clay containing organicrich layers (black shales). The event is marked regionally by a prominent seismic reflector and local unconformity, and seems to coincide with the initiation of seafloor spreading between Iberia and Newfoundland as determined from magnetic anomalies.

Drilling also confirms the presence of serpentinized peridotite, which is probably altered rock from the upper mantle, on a long ridge close to the boundary between oceanic and continental crust. This ridge might be a fragment of Palaeozoic (300 - 500 Myr) basement, or it may have been emplaced during the last stages of the rifting process as a result of thinning of the crust. The changes in sedimentary regime accompanying this rifting are very clearly documented by the drilling results from Leg 103, which have also revealed the hazards of geologic interpretation based solely on geophysics.

*Co-chiefs scientists: G. Boillot (Univ. Pierre et Marie Curie, France) and E. L. Winterer, (Scripps Institution o Oceanography). Also J. Applegate (Florida State Univ.), M Baltuck (Tulane Univ.), J. A. Bergen (Florida State Univ.), M Comas (Univ. de Granada, Spain), T. A. Davies (Univ. of (Cxas, Austin), K. Dunham (Univ. or Michigan), C. A. Evans Colgate Univ.) J. Girardeau (Institur de Physique du Giobe Obser. D. (Bedford In), Univ of California Los Angeles), Kasahara (Earthquake Research Institute, Univ, of Tokyo), J.-P. Loreau (Museum Research Institute, Univ. of Tokyo), J.-P. Loreau (Museum (Hispanoil-Eniepsa Madrid, Spain), A. W. Meyer ODP, Texas A\&M Univ.), M. Moullade (Univ. de Nice France), J. Ogg (Scripps), M. Sarti (Univ. di Ferrara, Italy), J. Thurow (Univ. Tübingen, F.R.G.), M. W. Williamson (Dalhousie Univ.).

\title{
Supernovae
}

\section{Little Bear's mass loss rate}

\section{from Virginia Trimble}

WhICH STARS explode as supernova, and how do they do it? Astronomers have debated these questions since Walter Baade and Fritz Zwicky identified and defined supernovae as a distinct astronomical phenomenon in 1934'. Three recent contributions ${ }^{24}$ seem to have resolved at least one facet of this problem. The current consensus is that Tpe 1 (hydrogen free) events arise in relatively old, low-mass stars and derive energy from explosive burning of carbon and oxygen. Type 11 (hydrogen-rich) supernovae, on the other hand, end the lives of young, massive stars, whose cores collapse to neutron star densities or beyond. The light we see comes from a shock wave propagating through many solar masses of hydrogen in an envelope $10^{14}-10^{15} \mathrm{~cm}$ across. Massive stars develop such envelopes during their late, red supergiant evolutionary phases.

Without these envelopes, broad-peaked light curves cannot be reproduced by the models ${ }^{5.6}$. And herein lies the main discrepancy with the present majority view that all or most single and wide binary $O B$ stars shed their extended, hydrogen-rich envelopes, becoming, first, Of stars, and then Wolf-Rayet (WR) stars before exploding $^{7-4}$. There could still be enough hydrogen $\left(0.1-0.3 M_{\odot}\right)$ to produce the spectra! lines we see, but the exploding stars wili be compact. As a result, most of the shock energy goes into expanding the star rather than into radiation, yielding a very dim supernova. And if there is too little hydrogen in the envelope, its recombination cannot produce the prolonged plateau phase seen in most Type III light curves.

The first of the new contributions comes from Schild and Maeder', who have looked again at the numbers of $\mathrm{OB}$ stars versus numbers of WR stars. They conclude that only stars in excess of $18 M_{\odot}$ give rise to nitrogen-rich WRs and only those above $35 M_{\odot}$ make the carbon-rich ones. In addition, they find that most $18-40 M_{\odot}$ stars need never go through a WR phase at all. This permits a reasonable range of intermediate mass stars to retain their extended, hydrogen-rich supergiant envelopes and make Type II light curves and spectra. One implication is that if the progenitor of the Crab Nebula, at $9 \pm 1$ $M_{\odot}$, stripped most of its envelope before exploding, it was unusual in doing so. This is good; it helps explain the rarity of pure, filled-centre remnants.

Second, Niemala, Ruiz and Phillips ${ }^{3}$ have, through a combination of luck and preparedness, managed to catch the Type Il event $1983 \mathrm{k}$ in NGC4699 nearly 10 days before maximum light. The pre-maximum spectrum shows $\mathrm{N}$ ॥ lines superimposed on a strong blue continuum, suggesting a progenitor whose surface composition resembled that of a WR star. Near maximum light the emission lines disappear, leaving weak $\mathrm{H}, \mathrm{He} \mathrm{and}$ Ca $n$ absorption lines. A month after maximum, the spectra are dominated by the P Cygni emission lines of $\mathrm{H}$ I that characterize normal Type II supernovae. The light curve is also quite normal. The implication is that we were seeing, first, emission from photospheric material as the shock wave emerges from the stellar core, then absorption in a normal-composition circumstellar envelope (shed by the star prior to core collapse), and finally, emission lines from the hydrogen-rich zone when the shock reaches and heats it. The light curve has a very broad peak, implying a very extended, pre-existing circumstellar shell.
Niemala et al. conclude that $1983 \mathrm{k}$ most likely resulted from a massive star that retained five or more solar masses of hydrogen-rich envelope but had also lost 1-2 $M_{\odot}$ into a circumstellar shell before exploding. The surface layers contained nitrogen-rich material, but the authors do not insist that the star would have looked like a WR if observed pre-explosion. They consider it equally possible that the WRlike lines they see are a result of the physical conditions produced by the supernova event itself. One last surprise: supernova 1983k occured $17(75 / H) \mathrm{kpc}$ from the centre of its parent galaxy, in projection, and well away from any $\mathrm{H}$ in regions or spiral arms that could be seen in a deep charge coupled device plate of the galaxy. The progenitor must, therefore, have been a runaway star or have been formed well away from the usual spiral arm sites.

The third contribution is from Josef Shklovskii, who tied several ideas together shortly before his death to provide an explanation of the absence of Type II supernovae in Magellanic irregular galaxies ${ }^{4}$. This absence has long been a puzzle, because these galaxies are the richest of all in gas and young stars. Yet only Type I supernovae have been detected in them. Shklovskii's explanation is that massive stars in these galaxies never develop the extended stellar or circumstellar envelopes needed to make Type II light curves as a direct result of their low metal abundances. Radiation passing through a star needs the absorption lines of heavy elements in order to transfer momentum to the outer layers and drive them off in a wind. Thus, massive stars in irregular galaxies never expand properly and, like stars that have been completely stripped, produce very dim supernovae. Shklovskii noted the progenitor of Cas A as a possible example of nonexpansion in our own Galaxy. I suspect complete stripping is also a possible interpretation for the faintness of that explosion.

The net conclusion we can draw from these studies ${ }^{2-4}$ is that the ideal Type II progenitor must have just the right amount of mass loss. Such stars occur in the Milky Way, NGC4699, and other spiral galaxies, but do not occur in elliptical or irregular galaxies.

1. Baade. W. \& Zwicky. F. Proc. natn. Acad. Sci 20.259 (1934); Phys. Rev. 45, 38, 46, 76 (3934).

Schild, H. \& Maeder, A. Astron. Astrophys. 136, 237 (1984).

3. Niemala, V.S., Ruiz, M.T. \& Phillips. M.M. Astrophys J. 289. 52 (1985)

4. Shklovskii. J.S. Sov. Astron. - AJ Lett. 10, 302 (1984)

Falk, S.W. \& Arnett. W.D. Astrophys. J. Suppl. 33, 515 (1977)

Woosley, S.E. \& Weaver, T.A. Preprint (proceedings of 1983 Santa Cruz workshop) 1985)

7. Conti, P., Garmany. C.. de Loore, C. \& Vanbeveren, D. Astrophys. J. 274. 302 (1983)

8. Falk, S.W. \& Mitalas. R. Mon. Nor. R. asir. Soc, 202, 19 (1983)

9. Berteili, G.. Bressan. A.G. \& Chiosi, C.S. Astron. Astro phys. 130. 278 (1984)

Virginia Trimble is Professor of Physics at the University of California, Irvine, California 92717, USA; and Visiting Professor of Astronomy at the University of Maryland, College Park, Maryland 20742, USA. 